\title{
PRINCIPAL HOMOGENEOUS SPACES FOR FINITE GROUP SCHEMES
}

\author{
STEPHEN S. SHATZ
}

S. U. Chase has proved [1] the following theorem:

Let $R$ be a commutative ring with unity, let $A$ be a finite, projective $R$ module which is also a cocommutative Hopf algebra over $R$ with antipode. If $\boldsymbol{X}(A)$ denotes the $R$-isomorphism classes of Galois $A$-objects over $R$, then there is a natural isomorphism

$$
X(A) \stackrel{\sim}{\sim} \operatorname{Ext}_{Z_{\mathrm{ar}}}^{1}\left(A^{D}, \mathrm{G}_{m}\right) .
$$

Here, a Galois $A$-object is exactly what geometers mean by a principal homogeneous space (PHS) for Spec $A$ over Spec $R$, the $\operatorname{Ext}_{\text {Zar }}^{1}$ refers to extensions of sheaves in the Zariski topology over $R$, and $A^{D}$ is the linear $R$-dual of $A$.

We wish to give a new, short proof of this theorem having two advantages: It applies even when $\operatorname{Spec} R$ is replaced by an arbitrary prescheme, and it explains why only the Zariski topology is needed. The price paid is that a certain amount of machinery is used, the proof we give being less explicit than the original one.

If $\pi: Y \rightarrow X$ is an affine morphism of preschemes, we shall say that $Y$ is locally a projective module over $X$ if and only if for every affine open $U$ in $X, \Gamma\left(\pi^{-1}(U), \theta_{Y}\right)$ is a projective $\Gamma\left(U, \theta_{X}\right)$ module. As an example, if $X$ is noetherian and $Y$ is finite and flat over $X$, then $Y$ is locally a projective module over $X$.

Theorem (ChASe). Let $X$ be a prescheme, and let $G$ be a finite commutative group scheme over $X$ which is locally a projective module over $X$. If $\mathbf{P H S}_{X}(G)$ denotes the group of (isomorphism classes of) PHS's for $G$ over $X$, then there is a natural isomorphism

$$
\operatorname{PHS}_{X}(G) \stackrel{\sim}{\sim} \operatorname{Extzar}^{1}\left(G^{D}, G_{m}\right)
$$

where Ext $\mathrm{Z}_{\mathrm{Zar}}^{1}$ refers to extensions of sheaves in the Zariski topology over $X$, and $G^{D}$ is the Cartier Dual of $G$.

Proof. There is the local-global spectral sequence for Ext in the flat topology on $X$ viz:

$$
H_{f l}^{p}\left(X, \operatorname{Ext}_{f l}^{q}\left(G^{D}, G_{m}\right)\right) \Rightarrow \operatorname{Ext}_{f l}^{*}\left(G^{D}, G_{m}\right) .
$$


Since $G^{D}$ is finite over $X$, the sheaf $\operatorname{Ext}_{f l}\left(G^{D}, G_{m}\right)$ is trivial for all $q>0^{1}$; hence, the spectral sequence collapses and we obtain isomorphisms

$$
H_{f l}^{p}(X, G) \stackrel{\sim}{\longrightarrow} \operatorname{Ext}_{f l}^{p}\left(G^{D}, G_{m}\right) .
$$

(Here, we have used the autoduality of $G$, i.e., $G^{D D}=G$.) When $p=1$, the group on the left in $\left({ }^{*}\right)$ is precisely $\operatorname{PHS}_{\boldsymbol{X}}(G)$ as is well known. Therefore, it suffices to prove that the natural injection

$$
\operatorname{Ext}_{\mathrm{zar}}^{1}\left(G^{D}, G_{m}\right) \rightarrow \operatorname{Ext}_{f l}^{1}\left(G^{D}, G_{m}\right)
$$

is an isomorphism. ${ }^{2}$ But a flat extension of $G_{m}$ by $G^{D}$ yields a locally trivial (in the flat topology) principal $G_{m}$-bundle over $G^{D}$; hence, a line bundle over $G^{D} .^{3}$ As is well known, every line bundle over $G^{D}$ may be split in a Zariski open covering, and this shows that our flat extension is in reality a Zariski extension, as contended. Q.E.D.

Remark. The same spectral sequence with $G^{D}$ replaced by $A^{t}-$ the dual of a projective abelian scheme $A$ over $X$-yields two facts:

$$
\operatorname{Ext}^{1}\left(A^{t}, G_{m}\right)(S)=\operatorname{Ext}_{S}^{1}\left(A^{t} \times S, G_{m} \times S\right)
$$

for any $S$ over $X$; i.e., $S \rightarrow \operatorname{Ext}_{S}^{1}\left(A^{t} \times S, G_{m} \times S\right)$ is already a sheaf, not just a presheaf.

$$
\operatorname{PHS}_{X}(A) \stackrel{\sim}{\longrightarrow} \operatorname{Ext}_{f l}^{2}\left(A^{t}, G_{m}\right),
$$

so that the proper way of viewing the duality pairings of Tate and others

$$
H^{0}(X, A) \otimes \operatorname{PHS}_{X}(A) \rightarrow \operatorname{Br}(X), \text { etc., }
$$

is via the Yoneda product. Similar remarks have been made by $M$. Artin and others including Tate himself.

AdDEd IN PRoof. W. Waterhouse (oral communication) has independently considered Chase's Theorem from the above point of view, and will publish his own (somewhat more detailed) account of it shortly.

Received by the editors July 17, 1968.

1 To prove this, one may refer to Oort [2, Proposition II. 8-4] and make the appropriate sheafifications, or apply the theory of SGAD, specifically the éxposés (6 and 7) of Gabriel.

${ }^{2}$ I wish to acknowledge here my indebtedness to both D. G. Quillen and S. U. Chase for incisive conversations on this point. [5].

3 For these facts, the reader may refer to Rosenlicht [3], and Grothendieck [4], 


\section{REFERENCES}

1. S. U. Chase, Galois theory and extensions of Hopf algebras, Lecture Notes in Math, Proc. Battelle Summer 1968 Conference, Springer-Verlag, Berlin (to appear).

2. F. Oort, Commutative group schemes, Lecture Notes in Math., No 15, SpringerVerlag, Berlin, 1966.

3. M. Rosenlicht, Some basic theorems on algebraic groups, Amer. J. Math. 78 (1956), 401-443.

4. A Grothendieck, Seminaire gémétrie algebrique, Inst. Hautes Etudes Sci. Publ. Math., Paris, 1960-1961.

5. - Techniques de construction et théorèmes d'existence en géométrie algébrique. III, Seminaire Bourbaki, Exposé 212, 1960-1961.

University of Pennsylvania 